\title{
Perfil antipsicótico do canabidiol
}

\section{Antipsychotic profile of cannabidiol}

\author{
João Francisco Cordeiro Pedrazzi', Ana Carolina de Castro Issy Pereira², Felipe Villela Gomes³, \\ Elaine Del Bel ${ }^{4}$
}

\begin{abstract}
RESUMO
O canabidiol, fitocanabinóide presente na planta Cannabis sativa é desprovido dos efeitos psicotomiméticos característicos do principal composto da Cannabis, o $\Delta^{9}$-tetraidrocanabinol, mais conhecido como delta 9-THC. Um conjunto crescente de evidências sugere que o canabidiol apresente potencial terapêutico para o tratamento dos sintomas de distúrbios psiquiátricos, como a depressão, a ansiedade e as psicoses. A observação em humanos, mas também em modelos animais experimentais da capacidade do canabidiol de antagonizar os efeitos psicotomiméticos do delta 9-THC constitui uma importante evidência de seu potencial para utilização clínica. Embora os efeitos farmacológicos do canabidiol tenham sido investigados em diferentes sistemas biológicos in vitro e in vivo, seu mecanismo de ação ainda não é claro. O delta 9-THC ativa os receptores canabinóides do tipo CB1 e CB2, contudo o canabidiol apresenta uma baixa afinidade por esses receptores. Adicionalmente, o canabidiol tem apresentado boa tolerabilidade em testes com humanos, tornando-o alvo de grande interesse da comunidade científica. O objetivo dessa revisão é apresentar, de forma breve, algumas das principais evidências experimentais e clínicas do provável perfil antipsicótico do canabidiol.
\end{abstract}

Palavras-chave: Canabidiol. Esquizofrenia. Antipsicóticos. Modelos Experimentais.

\section{Introduçåo}

O canabidiol (CBD) é um dos principais fitocanabinóides presentes na planta Cannabis sativa e diferente do seu principal constituinte, o $\Delta^{9}$-tetraidrocanabinol (delta-9-THC), é desprovido de efeitos psicotomiméticos. O CBD foi isolado no início da década de $40^{1}$, contudo, sua estrutura química foi elucidada apenas na década de 60 por Raphael Mechoulam e colaboradores. $^{2}$ Ao longo dos anos, o grupo do pesquisador Mechoulam foi responsável pela determina- ção da estrutura e das características estereoquímicas dos principais canabinóides presentes na Cannabis sativa, incluindo o CBD, o que incentivou a pesquisa da atividade farmacológica desses compostos.

A observação de que o CBD poderia antagonizar alguns dos principais efeitos farmacológicos do delta-9-THC levou a hipótese de que o CBD poderia apresentar uma ação ansiolítica, bem como também um perfil antipsicótico. ${ }^{3}$ Essa hipótese continua sendo testada em diferentes modelos animais. ${ }^{4,5}$ Esse trabalho apresenta objetivamente o sistema endocanabinói-
1. Mestrando pelo Departamento de Neurociências e Ciências do Comportamento da Faculdade de Medicina de Ribeirão Preto/ USP;

2. Pós-Doutoranda pelo Departamento de Morfologia, Fisiologia e Patologia Básica da Faculdade de Odontologia de Ribeirão Preto-FORP/USP

3 Doutorando pelo Departamento de Farmacologia da Faculdade de Medicina de Ribeirão Preto /USP.

4. Professora Titular no Departamento de Morfologia, Fisiologia e Patologia Básica da - FORP/USP.
Correspondência: Profa. Dra. Elaine Del Bel. Departamento de Morfologia, Fisiologia e Patologia Básica da Faculdade de Odontologia de Ribeirão Preto - FORP/USP. Avenida do Café S/№, 14040-904, Ribeirão Preto, SP, Brasil.

Artigo recebido em 16/10/2013 Aprovado para publicação em 04/02/2014 
de, as propriedades farmacológicas do CBD em modelos experimentais e em alguns estudos clínicos, além de descrever as expectativas do uso desse composto no tratamento dos sintomas da esquizofrenia.

\section{Neurobiologia do sistema endo- canabinóide}

Na década de 90, a descoberta dos receptores canabinóides em ratos, denominados do tipo 1 (CB1) e localizados no sistema nervoso central $(\mathrm{SNC})^{6}$ e do tipo $2(\mathrm{CB} 2)^{7}$ a princípio localizados fora do $\mathrm{SNC}$, promoveu grande avanço na compreensão dos efeitos da Cannabis sativa. O delta-9-THC, principal constituinte psicotrópico da Cannabis sativa se liga aos receptores CB1 e CB2. ${ }^{8}$ A descoberta dos receptores canabinóides trouxe a tona questões importantes, como quais seriam os ligantes endógenos para tais receptores e se canabinóides não psicotrópicos constituintes da Cannabis sativa, como por exemplo, o canabidiol, também se ligariam a receptores específicos. O provável mecanismo de ação do CBD será descrito a seguir.

\subsection{Os receptores canabinóides}

Os receptores CB1 localizam-se principalmente pré-sinapticamente no SNC, sendo encontrados em alta densidade no bulbo olfatório, hipocampo e cerebelo. Esses receptores apresentam densidade moderada no prosencéfalo e alguns núcleos do tronco cerebral e medula espinhal, áreas do córtex cerebral, amígdala e hipotálamo ventromedial. Por sua vez, uma baixa densidade desses receptores é observada no tálamo e em órgãos e tecidos periféricos. ${ }^{9,10}$ Observa-se que os receptores CB1 estão localizados em áreas associadas ao controle motor, aprendizagem e memória, resposta emocional e funções cognitivas, são ainda responsáveis pela maioria dos efeitos psicotrópicos dos canabinóides. ${ }^{11,12}$ A ativação dos receptores CB1 parece exercer papel fundamental nas experiências psicóticas agudas associadas ao uso da Cannabis sativa. ${ }^{13}$

$\mathrm{O}$ receptor $\mathrm{CB} 2$ é expresso, sobretudo, no sistema imunológico, contudo, sua presença é descrita em áreas específicas do SNC como, por exemplo, na microglia e em localização pós-sináptica. ${ }^{12}$ Estudos recentes sugerem que o receptor CB2 também pode ser expresso em células neurais envolvidas com a percepção/modulação da dor. ${ }^{14,15}$ Em modelos animais de dor, como a ligação do nervo espinhal e lesão por constrição crônica, foi descrito que o agonismo seletivo de receptores CB2 exerceu papel antinociceptivo. ${ }^{16-}$ 19

Os receptores CB1 e CB2 estão acoplados a uma proteína $\mathrm{G}$ inibitória que, quando ativada, inibe a enzima adenilato ciclase, levando a diminuição dos níveis de AMP cíclico e a inibição de canais de cálcio. ${ }^{20}$ $A$ ativação dos receptores CB1 inibe a liberação de outros neurotransmissores, inibitórios ou excitatórios, como o GABA e o glutamato. A descoberta dos receptores canabinóides desencadeou uma intensa busca pelos seus ligantes endógenos.

\subsection{Agonistas endógenos dos receptores canabinóides}

Os principais agonistas endógenos dos receptores CB1 e CB2 são os derivados do ácido araquidônico, que assim como os canabinóides presentes na Cannabis sativa são de natureza lipídica. Os endocanabinóides mais estudados são a anandamida (araquidonoil etanolamina) e o 2-araquidonoil-glicerol (2-AG), embora outros compostos endógenos já tenham sido descritos. ${ }^{21}$

A ativação e a biodisponibilidade dos endocanabinóides são controladas por diferentes enzimas. Diferente dos neurotransmissores clássicos, os endocanabinóides não são sintetizados nos terminais pré-sinápticos ou armazenados em vesículas ${ }^{5,22}$, mas sintetizados de acordo com a demanda nos terminais póssinápticos. ${ }^{20}$ Após o influxo de cálcio, induzido por glutamato ou GABA, ocorre a ativação de fosfolipases que convertem os fosfolipídios em endocanabinóides que atuam nos terminais pré-sinápticos onde inibem a liberação desses neurotransmissores. ${ }^{8}$ Esses compostos parecem atingir imediatamente a fenda sináptica por meio da difusão livre e ativam receptores CB1 présinápticos. ${ }^{23}$ Como mensageiros sinápticos retrógrados os endocanabinóides medeiam a transferência das informações dos terminais pós aos pré-sinápticos e são classificados como neurotransmissores atípicos em contraposição aos neurotransmissores clássicos. ${ }^{20,21}$

A disponibilidade dos endocanabinóides é regulada por captação-degradação enzimática. De forma bastante eficiente, as enzimas amida hidrolase de ácidos graxos e a monoacilglicerol lipase, respectivamente, hidrolisam a anandamida e o 2-AG. ${ }^{21,24}$

Devido a sua ampla distribuição no SNC é sugerido que endocanabinóides participem da modulação de uma variedade de funções cerebrais, incluindo memória e aprendizado, funções executivas e proces- 
sos motivacionais. ${ }^{22}$ Os avanços na compreensão da neurobiologia do sistema endocanabinóide, o que também amplia o conhecimento dos ligantes exógenos desse sistema, são fonte de otimismo na busca de novos agentes terapêuticos.

\section{Mecanismo de ação do CBD}

Embora o mecanismo de ação do CBD não seja completamente conhecido, é provável que o CBD não atue em receptores específicos tal como o delta-9THC. O CBD é capaz de facilitar a sinalização dos endocanabinóides através da inibição da recaptação ou hidrólise enzimática da anandamida, porém, em oposição ao delta-9-THC apresenta baixa afinidade aos receptores CB1 e CB2.25 Além disso, o CBD parece apresentar propriedades agonísticas nos receptores serotonérgicos do tipo 5-HT1A,,${ }^{9,26}$ pode ativar um canal iônico denominado receptor vanilóide do tipo 1 (TRPV1 $)^{27}$ e aumentar a sinalização mediada pela adenosina através da inibição de sua recaptação ${ }^{28}$. No sistema nervoso central, o TRPV1 é expresso nos terminais nervosos pós-sinápticos e é ativado pela anandamida. ${ }^{29}$ Entretanto, o envolvimento desses mecanismos nas propriedades antipsicóticas do CBD necessitam ser testados. É sugerido que a propriedade antipsicótica do CBD esteja relacionada à sua habilidade de aumentar a disponibilidade de anandamida. ${ }^{30}$

\section{Primeiras evidências do provável perfil antipsicótico do CBD}

A esquizofrenia é um transtorno psiquiátrico de etiologia multifatorial envolvendo variáveis genéticas, modificações neuroquímicas e alterações no desenvolvimento do sistema nervoso central. A pluralidade de suas manifestações promove uma patologia de quadros distintos que atinge cerca de $1 \%$ da população mundial. ${ }^{31,32}$ A esquizofrenia é caracterizada por três grandes grupos de sintomas, classificados como: positivos, negativos e cognitivos. ${ }^{33,34}$ Os sintomas positivos seriam fenômenos mentais ausentes em indivíduos sadios e são caracterizados principalmente por alucinações e ideias delirantes. O termo sintomas negativos é utilizado para descrever a perda da motivação, comprometimento social e embotamento afetivo. ${ }^{33} \mathrm{Os}$ sintomas cognitivos afetam funções como a atenção, memória e funcionamento intelectual geral. ${ }^{35}$

Os antipsicóticos são classificados em típicos e atípicos, sendo que a distinção entre esses dois tipos nem sempre é clara, mas essencialmente leva em consideração a afinidade desses compostos pelos receptores D2 e consequente risco de indução de efeitos motores indesejáveis. Assim, os antipsicóticos típicos são os que têm elevada afinidade pelos receptores D2 e, com frequência, produzem efeitos motores graves, como por exemplo, o Parkinsonismo farmacológico e a discinesia tardia. ${ }^{36}$ Os antipsicóticos atípicos apresentam menor afinidade pelos receptores D2 e menor probabilidade de promover efeitos motores adversos. Os antipsicóticos atuam ainda em outros sistemas neurotransmissores e sua eficácia no tratamento dos diferentes sintomas da esquizofrenia não é homogênea. ${ }^{37}$

A exposição ao delta 9-THC pode causar sintomas psicóticos em indivíduos sadios e em portadores de esquizofrenia. ${ }^{38}$ Nesses últimos pode haver ainda piora dos sintomas já existentes. ${ }^{38}$ A primeira evidência de que o CBD poderia apresentar propriedades do tipo antipsicótica foi obtida pela observação de que os efeitos subjetivos desencadeados pelo delta 9THC em voluntários sadios poderiam ser inibidos pelo CBD. ${ }^{39,40,41}$ Os efeitos induzidos pelo delta 9-THC eram semelhantes aos observados nas psicoses como a desfragmentação do pensamento, alterações da percepção e resistência à comunicação.

Além da relação dos canabinóides exógenos, como o delta 9-THC, e a psicose, uma série de estudos foi conduzida para explorar o papel dos endocanabinóides na neurobiologia da esquizofrenia. Objetivamente, grande parte desses estudos revelou níveis aumentados dos endocanabinóides no líquido cefalorraquidiano e sangue dos portadores de esquizofrenia $^{3,42,43}$ (para revisão Schubart et al., 2011). Nesse contexto, foi descrito em pacientes esquizofrênicos que apresentavam maiores concentrações de anandamida no líquido cefalorraquidiano em comparação aos controles saudáveis ${ }^{24}$, que essas concentrações estavam correlacionadas negativamente com os sintomas psicóticos, o que sugere que o aumento de anandamida envolva uma resposta de feedback negativo afim de contrabalançar os sintomas psicóticos e a hiperativação dos receptores D2. ${ }^{3}$ O CBD tem mostrado efeitos promissores em modelos animais preditivos para ação de drogas antipsicóticas, assim como em ensaios clínicos.

\subsection{CBD em modelos animais}

Grande parte dos efeitos do CBD em modelos animais foi obtida em preparações que empregam fármacos que alteram a neurotransmissão dopaminérgica, 
considerando o papel-chave desse neurotransmissor nas psicoses. ${ }^{44}$ Foi demonstrado que tanto o CBD como o antipsicótico típico haloperidol são capazes de atenuar a estereotipia induzida pelo agonista dopaminérgico apomorfina, de maneira dose dependente. ${ }^{45}$ Os mesmos autores demonstraram que a catalepsia, modelo animal do efeito adverso motor induzido por antipsicóticos típicos foi observada apenas nos animais tratados com haloperidol, mas não nos animais tratados com CBD, mesmo em altas doses $(480 \mathrm{mg} /$ $\mathrm{kg}){ }^{45}$ Esses achados foram corroborados pelo trabalho de Moreira e Guimarães (2005) ${ }^{46}$, que demonstraram que tanto o CBD quanto os antipsicóticos típico e atípico, haloperidol e clozapina, revertem a hiperlocomoção induzida por anfetamina, mas apenas o haloperidol induz catalepsia. Essa característica sugere que o CBD apresente perfil neurofarmacológico semelhante aos antipsicóticos atípicos. ${ }^{45,46}$ Adicionalmente, a administração crônica de CBD também atenua a hiperlocomoção induzida por anfetamina em camundongos. ${ }^{47}$ Em estudo de Malone e colaboradores, o CBD foi capaz de reverter à diminuição da interação social em ratos causada por baixas doses de delta 9-THC. ${ }^{48}$ Os modelos animais sugerem que o delta 9-THC aumenta a liberação de dopamina na via dopaminérgica mesolímbica $^{3}$ (para revisão Markus et al., 2008).

Uma vez que a dopamina exerce controle negativo sobre a secreção do hormônio prolactina, os antipsicóticos, que atuam como antagonistas de dopamina podem aumentar a secreção desse hormônio. ${ }^{2}$ Apesar de tanto o haloperidol quanto o CBD aumentarem os níveis plasmáticos de prolactina, o CBD é menos potente, induzindo aumentos significativos apenas após doses de 120 e $240 \mathrm{mg} / \mathrm{kg} .{ }^{49}$

$\mathrm{O} C B D$, assim como o antipsicótico atípico clozapina, aumenta a expressão da proteína c-Fos no núcleo acumbens, mas não em regiões motoras como o estriado dorsal, característica dos antipsicóticos típicos como o haloperidol, provavelmente relacionada com as manifestações motoras adversas dessa classe de antipsicóticos. ${ }^{50}$ A proteína c-FOS é codificada por um gene de indução rápida e transiente e é utilizada como uma medida funcional de ativação neuronal. ${ }^{10}$ Essa observação indica que o CBD produz ativação neuronal na via mesolímbica e provavelmente, menor potencial para os efeitos motores indesejáveis, mais uma vez corroborando o provável perfil atípico desse composto. ${ }^{41}$

O perfil antipsicótico do CBD foi estendido a um modelo baseado no sistema glutamatérgico. $\mathrm{O}$
CBD inibe a hiperlocomoção causada por ketamina um antagonista do receptor $\mathrm{NMDA}^{46}$, além daquela produzida por anfetamina, de forma semelhante aos antipsicóticos haloperidol e clozapina. Em concordância com essas observações, o CBD, semelhante à clozapina, reverte o prejuízo no teste de inibição pré-pulso (prepulse inhibition - PPI) em camundongos e a hiperatividade e a redução da interação social em ratos, provocados por MK-801, outro antagonista do receptor NMDA. ${ }^{51,52} \mathrm{O}$ efeito do $\mathrm{CBD}$ na atenuação do prejuízo no teste de PPI induzido pelo MK-801 foi bloqueado pelo tratamento com capzasepina, um antagonista do receptor TRPV1. ${ }^{25}$ Antipsicóticos típicos, em contrapartida, geralmente são incapazes de restaurar déficits no teste de PPI e na interação social induzidos por antagonistas do receptor NMDA. ${ }^{23,53}$

A tabela 1 apresenta sumário de resultados obtidos com o CBD em modelos animais de manifestações positivas da esquizofrenia.

\subsection{CBD na clínica}

Pesquisas envolvendo o CBD na clínica também evidenciam seu potencial antipsicótico. ${ }^{41}$ A pesquisa clínica do uso do CBD no tratamento dos sintomas da esquizofrenia teve início no Brasil em meados de $1990 .{ }^{54}$ Portadores de esquizofrenia expostos ao CBD apresentaram considerável melhora de seus sintomas psicóticos como, por exemplo, pensamentos desconexos e distúrbios de percepção, sintomas positivos da esquizofrenia ${ }^{3}$ (para revisão Zuardi et al., 2013). De forma interessante, os efeitos colaterais comuns aos antipsicóticos, como os efeitos extrapiramidais, não são provocados pelo CBD. ${ }^{36} \mathrm{Em}$ indivíduos saudáveis é observado que o CBD atenua o prejuízo no tempo de execução de tarefas e na euforia, condições induzidas pelo delta-9-THC. ${ }^{55,56}$ Corroborando essas observações, foi observado que pacientes de um hospital psiquiátrico na África do Sul, após a utilização de uma variedade de Cannabis praticamente desprovida de CBD apresentaram uma maior frequiência de episódios psicóticos agudos, o que de forma hipotética, sugere que a presença de CBD em amostras de Cannabis sativa protege seus usuários contra a ocorrência de episódios psicóticos que podem ser induzidos pelo delta-9-THC. ${ }^{56}$

Recentemente, resultados de um estudo clínico controlado com 42 pacientes esquizofrênicos ou com psicose esquizofreniforme comparando os efeitos do tratamento por 4 semanas com CBD aos da amisul- 
Tabela 1: Sumário de alguns resultados obtidos em modelos animais com CBD.

\begin{tabular}{lllll}
\hline Estudo & Espécie & Dose CBD & Resultados & Referências \\
\hline $\begin{array}{l}\text { Estereotipia induzida } \\
\text { por apomorfina }\end{array}$ & ratos & $60 \mathrm{mg} / \mathrm{kg}$ & Redução & Zuardi et al.(1991) \\
$\begin{array}{l}\text { Expressão da proteína c-Fos } \\
\text { ratos }\end{array}$ & $120 \mathrm{mg} / \mathrm{kg}$ & $\begin{array}{l}\text { Aumento no } \\
\text { núcleo acumbens }\end{array}$ & Guimarães et al. (2004) \\
$\begin{array}{l}\text { Hiperlocomoção induzida } \\
\text { por anfetamina }\end{array}$ & camundongos & $30-60 \mathrm{mg} / \mathrm{kg}$ & Redução & Moreira e Guimarães (2005) \\
$\begin{array}{l}\text { Prejuízo no teste de } \\
\text { PPI induzido por MK-801 }\end{array}$ & camundongos & $5 \mathrm{mg} / \mathrm{kg}$ & Redução & Long et al.(2006) \\
\hline
\end{tabular}

prida, um antipsicótico atípico, mostraram que ambos os tratamentos foram igualmente eficazes em reduzir os sintomas psicóticos. Além disso, comparado a amisulprida, o CBD causou menor incidência de efeitos extrapiramidais, aumento nos níveis de prolactina e ganho de peso. ${ }^{57}$ Curiosamente, nesse mesmo estudo, foi observado que os pacientes tratados com CBD apresentaram maiores níveis séricos de anandamida, associado com a melhora dos sintomas clínicos. Dessa maneira, se a anandamida participa do efeito antipsicótico do CBD, a compreensão dos circuitos neuronais envolvidos nesse efeito ainda não estão claros.

Estudo conduzido por Bhattacharyya e colaboradores $(2012)^{58}$ utilizando medida de ativação cerebral em voluntários sadios, recentemente demonstrou que o delta-9-THC e o CBD diferentemente modulam a função de regiões cerebrais envolvidas no processamento de estímulos salientes. A ativação do córtex pré-frontal pelo delta-9-THC é responsável por alguns dos déficits cognitivos induzidos por esse composto. Adicionalmente, os efeitos do delta-9-THC sobre os sintomas psicóticos podem estar relacionados à ativação do estriado. Esse grupo demonstrou que o CBD exerce efeitos opostos aos do delta-9-THC sobre a ativação do estriado, córtex pré-frontal e córtex temporal medial. ${ }^{58}$ Esses mecanismos podem contribuir com os efeitos da Cannabis sativa sobre os sintomas psicóticos e com a habilidade do CBD em antagonizar os efeitos do delta-9-THC.

\section{Vantagens da utilização clínica do CBD}

Outro aspecto positivo acerca da possível utilização terapêutica do CBD é que existem diversos estudos in vivo e in vitro da administração do CBD sob um amplo espectro de concentrações, que não detectaram importantes efeitos colaterais e tóxicos. ${ }^{3}$ Além disso, a administração aguda de CBD por diferentes vias não induziu qualquer efeito tóxico significativo em humanos. Também foi observado que a administração crônica do CBD por um mês em voluntários sadios (doses diárias entre 10 a 400 mg), não promoveu nenhuma anormalidade em exames neurológicos, psiquiátricos ou clínicos. ${ }^{59}$ Embora haja relatos de alguns estudos, que o CBD possa induzir alguns efeitos colaterais menores, incluindo a inibição do metabolismo hepático da droga. De forma geral, os dados clínicos disponíveis sugerem que o CBD pode ser administrado com segurança em um amplo intervalo de doses, de forma semelhante aos resultados já encontrados em estudos animais. ${ }^{60}$

A tabela 2 apresenta sumário dos resultados obtidos com o CBD na clínica em estudos realizados em portadores de esquizofrenia.

\section{Considerações Finais}

O CBD é um componente da planta Cannabis sativa desprovido de propriedades psicotomiméticas. A descrição do sistema endocanabinóide permitiu o desenvolvimento de diferentes instrumentos farmacológicos que atuam nesse sistema. Essas drogas são ferramentas utilizadas em modelos animais que possibilitam a compreensão de como, e com que finalidade, esse sistema pode ser explorado pela indústria farmacêutica. Os modelos animais também auxiliam na elucidação do mecanismo de ação de drogas, como o CBD, e corroboram hipóteses acerca de seu potencial uso clínico. 
Tabela 2: Sumário de alguns dos resultados obtidos na clínica com CBD

\begin{tabular}{|c|c|c|c|c|}
\hline Estudo & Indivíduos & Dose CBD & Resultados & Referências \\
\hline Estudo de Caso & 1 indivíduo & $1200 \mathrm{mg} / \mathrm{dia}$ & Melhora dos sintomas & Zuardi et al. (1995) \\
\hline Estudo de Caso & $\begin{array}{l}3 \text { pacientes resistentes ao } \\
\text { tratamento convencional }\end{array}$ & $40-1280 \mathrm{mg} / \mathrm{dia}$ & $\begin{array}{l}\text { Melhora leve dos sin- } \\
\text { tomas em um paciente }\end{array}$ & Zuardi et al. (2006) \\
\hline Estudo de Caso & $\begin{array}{l}6 \text { pacientes com psicose } \\
\text { associada à doença de } \\
\text { Parkinson }\end{array}$ & $150 \mathrm{mg} / \mathrm{dia}$ & $\begin{array}{l}\text { Melhora clínica signifi- } \\
\text { cativa }\end{array}$ & Zuardi et al. (2009) \\
\hline Estudo de Caso & $\begin{array}{l}2 \text { pacientes com transtorno } \\
\text { bipolar }\end{array}$ & $600-1220 \mathrm{mg} / \mathrm{dia}$ & $\begin{array}{l}\text { Nenhuma melhora dos } \\
\text { sintomas }\end{array}$ & Zuardi et al. (2010) \\
\hline $\begin{array}{l}\text { Estudo duplo cego } \\
\text { randomizado } \\
\text { CBD x Amisulprida }\end{array}$ & $\begin{array}{l}22 \text { pacientes portadores de } \\
\text { esquizofrenia com paranoia } \\
\text { aguda }\end{array}$ & $\begin{array}{l}800 \text { mg de CBD } \\
\text { ou amisulprida }\end{array}$ & $\begin{array}{l}\text { Melhora clínica signifi- } \\
\text { cativa }\end{array}$ & $\begin{array}{l}\text { Leweke et al. } \\
\text { (2012) }\end{array}$ \\
\hline
\end{tabular}

O delta-9-THC e o CDB apresentam distinção clara de seus efeitos sobre diferentes parâmetros associados às psicoses. As variedades de Cannabis sativa que contêm maior concentração de CBD significativamente causam menor incidência de experiências do tipo psicóticas. ${ }^{43} \mathrm{O}$ CBD é capaz de atenuar os efeitos psicológicos induzidos pelo delta-9-THC em humanos e as alterações comportamentais induzidas em modelos animais. Resultados obtidos em modelos animais preditivos para efeito antipsicótico, ensaios clínicos, estudos baseados em análise de imagem e avaliações epidemiológicas corroboram a idéia do potencial perfil antipsicótico do $\mathrm{CBD}^{38,43}$ (para revisão Schubart et al., 2011).

Positivamente, foi observado que a administração do CBD, sob um amplo espectro de doses, não promove efeitos colaterais e tóxicos significativos. Até o momento, apenas um medicamento contendo CBD está disponível no mercado. O Sativex®, um spray bucal, que contém delta-9-THC e CDB (50/50\%) é recomendado como analgésico para uso em pacientes adultos portadores de câncer em estágios avançados e para o tratamento de espasticidade e dor neuropática em pacientes com esclerose múltipla. ${ }^{34,39}$ No entanto, o uso de Sativex não é recomendado para o tratamento dos sintomas da esquizofrenia devido à elevada proporção de delta-9-THC em sua composição.

\section{Agradecimentos}

Agradecemos ao professor Dr. Francisco Silveira Guimarães do Departamento de Farmacologia da Faculdade de Medicina de Ribeirão Preto, USP, pelas discussões sobre o tema abordado.

Conflitos de Interesse: os autores declararam não apresentar conflitos de interesse.

Suporte Financeiro: FAPESP 


\begin{abstract}
Cannabidiol an important phytocannabinoid present in the Cannabis sativa opposing to the major plant compound $\mathrm{D}^{9}$-tetrahydrocannabinol, known as delta-9-THC, is devoid of the psychotomimetic effects. Growing set of evidence suggest that cannabidiol may be used for the treatment of the symptoms of psychiatric disorders such as depression, anxiety and psychosis. The first evidence of the cannabidiol therapeutic potential was the observation of its ability to antagonize delta-9-THC effects either on human and experimental animal models. Pharmacological effects of CBD has been investigated in different biological systems, in vitro and in vivo, however, the mechanisms responsible for their therapeutic potential are still unclear. delta-9-THC effects results from activation of the cannabinoid receptors CB1 and CB2, however, the cannabidiol has low affinity for these receptors. The good tolerability of cannabidiol in human trials makes this compound an interesting target of the scientific community. The aim of this paper is to present concisely some experimental and clinical evidence about the cannabidiol antipsychotic profile.
\end{abstract}

Key words: Cannabidiol. Schizophrenia. Antipsychotic Agents. Experimental, Models.

\section{Referênclas blbllográficas}

1. Adams R, Hunt M, Clark JH. Structure of cannabidiol, a product isolated from the marihuana extract of Minnesota wild hemp. J Am Chem Soc. 1940; 62: 196-200.

2. Mechoulam R, Shvo Y. Hashish. I. The structure of cannabidiol. Tetrahedron. 1963; 19: 2073-8.

3. Zuardi AW, Crippa JA, Hallak JE, Bhattacharyya S, Atakan Z, Martin-Santos $R$ et al. A critical review of the antipsychotic effects of cannabidiol: 30 years of a translational investigation. Curr Pharm Des. 2012;18: 5131-40.

4. Campos AC, Guimarães FS. Involvement of $5 \mathrm{HT} 1 \mathrm{~A}$ receptors in the anxiolytic-like effects of cannabidiol injected into the dorsolateral periaqueductal gray of rats. Psychopharmacology. (Berl) 2008; 199: 223-30.

5. Daniel TM, Dennis J, David AT. Cannabidiol reverses the reduction in social interaction produced by low dose D9-tetrahydrocannabinol in rats. Pharmacol Biochem Behav. 2009; 93: 91-6.

6. Matsuda LA, Lolait SJ, Brownstein MJ, Young AC, Bonner TI. Structure of a cannabinoid receptor and functional expression of the cloned cDNA. Nature (Lond.). 1990; 346: 561-4.

7. Munro S, Thomas KL, Abu-Shaar M. Molecular characterization of a peripheral receptor for cannabinoids. Nature (Lond.). 1993; 365: 61-5.

8. Pertwee RG. The diverse $\mathrm{CB} 1$ and $\mathrm{CB} 2$ receptor pharmacology of three plant cannabinoids: delta9-tetrahydrocannabinol, canabidiol and delta9 tetrahydrocannabivarin. $\mathrm{Br} \mathrm{J}$ Pharmacol. 2008; 153: 199-215.

9. Russo EB, Burnett A, Hall B \& Parker KK. Agonistic properties of cannabidiol at 5-HT1a receptors. Neurochem Res. 2005; 30: 1037-43.

10. Morgan JI, Curran T. Fos: an immediate-early transcription factor in neurons. J Neurobiol. 1995; 26: 403-12.

11. Guindon J, Hohmann AG. The endocannabinoid system and pain. CNS Neurol Disord Drug Targets. 2009; 8: 403-21.

12. Sagar DR, Gaw AG, Okine BN, Woodhams SG, Wong A, Kendall DA et al. Dynamic regulation of the endocannabinoid system: implications for analgesia. Mol Pain. 2009; 5: 59.

13. Huestis MA, Gorelick DA, Heishman SJ, Preston KL, Nelson RA, Moolchan ET et al. Blockade of effects of smoked marijuana by the CB1-selective cannabinoid receptor antagonist SR141716. Arch Gen Psychiatry.. 2001; 58:322-8.
14. Zhang J, Hoffert C, Vu HK, Groblewski T, Ahmad S, O'Donnell D. Induction of CB2 receptor expression in the rat spinal cord of neuropathic but not inflammatory chronic pain models. Eur J Neurosci. 2003; 17:2750-4.

15. Elmes SJR, Jhaveri MD, Smart D, Kendall DA, Chapman V. Cannabinoid CB2 receptor activation inhibits mechanically evoked responses of wide dynamic range dorsal horn neurons in naý ve rats and in rat models of inflammatory and neuropathic pain. Eur J Neurosci. 2004; 20:2311-20.

16. Clayton N, Marshall FH, Bountra C, O'Shaughnessy CT. CB1 and CB2 cannabinoid receptors are implicated in inflammatory pain. Pain 2002; 96:253-60.

17. Ibrahim MM, Deng H, Zvonok A, Cockayne DA, Kwan J, Mata $\mathrm{HP}$ et al. Activation of CB2 cannabinoid receptors by AM1241 inhibits experimental neuropathic pain: pain inhibition by receptors not present in the CNS. Proc Natl Acad Sci U.S.A. 2003; 100: 10529-33.

18. Ibrahim MM, Porreca F, Lai J, Albrechtk PJ, Rice FL, Khodorova A et al. CB2 cannabinoid receptor activation produces antinociception by stimulating peripheral release of endogenous opioids. Proc Natl Acad Sci U.S.A. 2005; 102: 3093-8.

19. Nackley AG, Makriyannis A, Hohmann, AG. Selective activation of cannabinoid $C B 2$ receptors suppresses spinal fos protein expression and pain behavior in a rat model of inflammation. Neuroscience. 2003; 119: 747-57.

20. Piomelli, D. The molecular logic of endocannabinoid signaling. Nat Rev Neurosci. 2003; 11: 873-84.

21. Saito VM, Wotjak CT, Moreira FA. Pharmacological exploitation of the endocannabinoid system: new perspectives for the treatment of depression and anxiety disorders? Rev Bras Psiquiatr. 2010; 1: 7-14.

22. Di Marzo, V. Targeting the endocannabinoid system: To enhance or reduce? Nat Rev Drug Discov. 2008; 7: 438-55.

23. Geyer MA, Krebs-Thomson K, Braff DL, Swerdlow NR. Pharmacological studies of prepulse inhibition models of sensorimotor gating deficits in schizophrenia: a decade in review. Psychopharmacology. Berl 2001; 156: 117-54.

24. Giuffrida A, Leweke FM, Gerth CW, Schreiber D, Koethe D et al. Cerebrospinal anandamida levels are elevated in acute schizophrenia and are inversely correlated with psychotic symptoms. Neuropsychopharmacol. 2004; 29: 2108-14.

25. Mechoulam R, Hanus L. Cannabidiol: an overview of some chemical pharmacological aspects Chem Phys Lipids.. 2002; 121:35-43. 
26. Gomes FV, Reis DG, Alves FH, Correa FM, Guimarães FS, Resstel LB. Cannabidiol injected into the bed nucleus of the stria terminalis reduces the expression of contextual fear conditioning via 5-HT1A receptors. J Psychopharmacol. 2012; 26: 104-13.

27. Bisogno T, Hanus L, De Petrocellis L, Tchilibon S, Ponde DE, Brandi I. et al. Molecular targets for cannabidiol and its synthetic analogues: effect on vanilloid VR1 receptors and on the cellular uptake and enzymatic hydrolysis of anandamide. Br J Pharmacol. 2001; 134: 845-52.

28. Carrier EJ, Auchampach JA, Hillard CJ. Inhibition of an equilibrative nucleoside transporter by cannabidiol: a mechanism of cannabinoid immunosuppression. Proc Natl Acad Sci. U. S. A. 2006; 103: 7895-900.

29. Brown AJ. Novel cannabinoid receptors. Br J Pharmacol. 2007; 152: 567-75.

30. Schubart CD, Sommer IE, Fusar-Poli P, de Witte L, Kahn RS, Boks MP. Cannabidiol as a potential treatment for psychosis. Eur Neuropsychopharmacol. 2013; 11: 002.

31. Weiss SM, Feldon J. Environmental animal models for sensorimotor gating deficiencies in schizophrenia Psychopharmacology. 2001; 156: 305-26.

32. Lau YS, Petroske E, Meredith GE, John QW. Elevated neuronal NOS expression in chronic haloperidol-treated rats. Neuropharmacology. 2003; 45: 986-94.

33. Andreasen NC. Symptoms, signs, and diagnosis of schizophrenia. Lancet. 1995; 346: 477-81.

34. Wong $\mathrm{AH}$, Van Tol HH. Schizophrenia: from phenomenology to neurobiology. Neurosci Biobehav Rev. 2003; 27: 269-306.

35. Wong AH, Voruganti LN, Heslegrave RJ, Awad AG. Neurocognitive deficits and neurological signs in schizophrenia. Schizophr Res. 1997; 23: 139-46.

36 .Kane JM. Extrapyramidal side effects are unacceptable. Eur Neuropsychopharmacol. 2001; 4: 397-403.

37. Blin O. A comparative review of new antipsychotics. Can J Psychiatry. 1999; 44: 235-44.

38. Morrison PD, Nottage J, Stone JM ,Bhattacharyya S, Tunstall $\mathrm{N}$, Brenneisen $\mathrm{R}$ et al. Disruption of frontal è coherence by Ä9-tetrahydrocannabinol is associated with positive psychotic symptoms. Neuropsychopharmacol. 2011; 36: 82736 .

39. Karniol IG, Carlini EA. Pharmacological interaction between cannabidiol and delta 9 tetrahydrocannabinol. Psychopharmacologia. 1973; 33: 53-70.

40. Zuardi AW, Shirakawa I, Finkelfarb E, Karniol IG. Action of cannabidiol on the anxiety and other effects produced by delta 9-THC in normal subjects. Psychopharmacology. 1982 76: $245-50$

41. Zuardi AW. Cannabidiol: from an inactive cannabinoid to a drug with wide spectrum of action. Rev Bras Psiquiatr. 2008; 30: $271-80$

42. Koethe D, Giuffrida A, Schreiber D, Hellmich M, SchultzeLutter F, Ruhrmann S et al. Anandamide elevation in cerebrospinal fluid in initial prodromal states of psychosis. $\mathrm{Br} J$ Psychiatr. 2009; 194: 371-2.

43. Schubart CD, Sommer IE, vanGastel WA, Goetgebuer RL, Kahn RS, Boks MP. Cannabis with high cannabidiol content is associated with fewer psychotic experiences. Schizophr Res. 2011; 130: 216-21.
44. Howes OD, Kapur S. The dopamine hypothesis of schizophrenia: version III-the final common pathway. Schizophr Bull. 2009; 35: 549-62.

45. Zuardi AW, Rodrigues JA, Cunha JM. Effects of cannabidiol in animal models predictive of antipsychotic activity. Psychopharmacology. 1991; 104: 260-4.

46. Moreira FA, Guimarães FS. Cannabidiol inhibits the hyperlocomotion induced by psychotomimetic drugs in mice. Eur J Pharmacol. 2005; 512: 199-205.

47. Long LE, Chesworth R, Huang XF, McGregor IS, Arnold JC Karl T. A behavioural comparison of acute and chronic D9 tetrahydrocannabinol and cannabidiol in C57BL/6JArc mice. Int J Neuropsychopharmacol. 2010; 13: 861-76.

48. Malone DT, Jongejan D, Taylor DA. Cannabidiol reverses the reduction in social interaction produced by low dose Delta(9)tetrahydrocannabinol in rats. Pharmacol Biochem Behav. 2009; 93: 91-96.

49. Zuardi AW, Crippa JA, Hallak JE, Moreira FA, Guimarães FS. Cannabidiol, a Cannabis sativa constituent, as an antipsychotic drug. Braz J Med Biol Res. 2006: 39: 421-9.

50 .Guimarães VMC, Zuardi AW, Del Bel EA, Guimarães FS. Cannabidiol increases Fos expression in the nucleus accumbens but not in dorsal striatum. Life Sci 2004; 75: 633-8.

51. Gurujan A, Taylor DA, Malone DT. Cannabidiol and clozapine reverse MK-801-induced 3376 A. C. Campos et al. Review. Cannabidiol and psychiatric disorders deficits in social interaction and hyperactivity in Sprague-Dawle rats. J Psychopharmacol. 2012; 26: 1317-32.

52. Long LE, Malone DT, Taylor DA. Cannabidiol reverses MK 801-induced disruption of prepulse inhibition in mice. Neuropsychopharmacol. 2006; 31: 795-803.

53. Curzon P, Decker MW. Effects of phencyclidine (PCP) and (b)MK-801 on sensorimotor gating in CD-1 mice. Prog. Neuropsychopharmacol. Biol Psychiatr. 1998; 22: 129-46.

54. Zuardi AW, Loureiro SR, Rodrigues CR. Reliability, validity and factorial dimensions of the interactive observations scale for psychiatric inpatients. Acta Psychiatr Scand. 1995; 91 247-51.

55. Karniol IG, Shirakawa I, Kasinski N, Pfeferman A, Carlini EA. Cannabidiol interferes with the effects of delta 9-tetrahydrocannabinol in man. Eur J Pharmacol. 1974; 28: 172-7.

56. Rottanburg D, Robins AH, Ben-Arie O, Teggin A, Elk R. Cannabis-associated psychosis with hypomanic features. Lancet. 1982; 2: 1364-6.

57. Leweke FM, Piomelli D, Pahlisch F, Muhl D, Gerth CW, Hoyer C et al. Cannabidiol enhances anandamide signaling and alleviates psychotic symptoms of schizophrenia. Transcult Psychiatry. 2012; 2: 94.

58. Bhattacharyya S, Crippa JA, Allen P, Martin-Santos R, Borgwardt S, Fusar-Poli $P$ et al. Induction of psychosis by Ä9 tetrahydrocannabinol reflects modulation of prefrontal and striatal function duringattentional salience processing Arch Gen Psychiatry. 2012; 69: 27-36.

59. Cunha J, Carlini EA, Pereira AE, et al. Chronic administration of cannabidiol to healthy volunteers and epileptic patients. Pharmacology. 1980; 21: 175-85.

60. Bergamaschi MM, Queiroz RHC, Zuardi AW, Crippa JAS. Safety and Side Effects of Cannabidiol, a Cannabis sativa Constituent. Curr Drogas Saf. 2011; 6: 237-49. 